\title{
Enhancement of post trabeculectomy bleb formation by laser suture lysis
}

\author{
J Singh, RW D Bell, A Adams, C O’Brien
}

\begin{abstract}
Aims-To study the efficacy of laser suture lysis in the promotion of filtration and bleb formation during the early postoperative period after trabeculectomy.

Methods-This was a prospective study of 20 eyes from 20 patients undergoing trabeculectomy. Patients were entered into the study if, after a trial period of ocular massage, the intraocular pressure (IOP) was greater than $21 \mathrm{~mm} \mathrm{Hg} 48$ hours after surgery. Other criteria for inclusion were a deep quiet anterior chamber, no bleb formation, and a patent internal ostium on gonioscopy. Suture lysis was performed using a frequency doubled neodymium YAG (532 nm) laser and a Hoskins lens. The IOP was measured within 5 minutes of lysis and gentle ocular massage was employed if there was no spontaneous bleb formation. A further suture was lysed if at this stage there was still no bleb associated with a high IOP.
\end{abstract}

Results-Suture lysis was successful in all cases in the early postoperative period. The mean IOP before lysis was 33.8 (SD 8.9) $\mathrm{mm} \mathrm{Hg}$ and the IOP immediately after lysis was $13.2(7.9) \mathrm{mm} \mathbf{~ H g}$. Eighteen $(90 \%)$ patients had successfully controlled IOP (mean 14.1 (3.6) $\mathrm{mm}$ Hg) without medication after a mean follow up of 6 months.

Conclusion-Laser suture lysis is a safe and effective method of promoting filtration following trabeculectomy. It can avoid surgical manipulation of the scleral flap with its associated risks of bleb failure and thereby contributes to a successful operative outcome.

(Br F Ophthalmol 1996;80:624-627)

Partial thickness glaucoma surgery in the form of trabeculectomy was first described by Cairns in $1968^{1}$ and is currently the most popular surgical treatment for glaucoma. This is due to the lower incidence of postoperative complications in comparison with full thickness procedures, where excessive aqueous outflow can lead to anterior chamber flattening, prolonged hypotony, peripheral anterior synechiae, and cataract formation. ${ }^{2-4}$ The advantage of the full thickness filtering procedure is the lower intraocular pressure (IOP) achieved postoperatively, ${ }^{2-4}$ which is particularly relevant for eyes with advanced glaucoma. This potential benefit, however, is more than offset by the associated problems previously mentioned and therefore this technique has largely been aban- doned by most glaucoma surgeons. Trabeculectomy aims to promote a filtering conjunctival bleb where a scleral flap must be loose enough to permit aqueous outflow but taut enough to prevent post surgical ocular hypotony and shallow or flat anterior chambers.

Failure of bleb formation in the immediate postoperative period in the presence of a high IOP, a deep anterior chamber, and normal gonioscopy may be due to an overtight scleral flap. Management of tight scleral flaps can be achieved by surgical reoperation or by loosening preplaced releasable sutures. ${ }^{5}$ In this study we have looked at the effectiveness of enhancing conjunctival filtering blebs after trabeculectomy surgery by laser lysis of tight nylon scleral flap sutures.

\section{Patients and methods}

This is a consecutive series of 20 eyes from 20 patients with a mean follow up of 6 months (range 3-10 months). There were 13 females and seven males with a mean age of 67 years. Fourteen patients had chronic open angle glaucoma, four had chronic angle closure glaucoma, two had pseudoexfoliative glaucoma, one had secondary glaucoma following anterior uveitis, and there was one case of secondary glaucoma following penetrating keratoplasty for acanthamoeba keratitis.

All patients underwent trabeculectomy surgery by one of three surgeons. Fornix based conjunctival flaps were created followed by diathermy to the sclera before a rectangular scleral flap measuring $3 \times 3$ or $4 \times 4 \mathrm{~mm}$ was dissected to clear cornea. Fourteen cases had a paracentesis created before a block of corneoscleral tissue was excised. A peripheral iridectomy was performed and the scleral flap was sutured with two or three $10 / 0$ nylon stitches. The conjunctiva was closed with buried nylon sutures. A subconjunctival injection of $20 \mathrm{mg}$ gentamicin was given at the end of the procedure. There were three patients who had their drainage surgery augmented with peroperative administration of 5-fluorouracil (5FU): these included the two cases of secondary glaucoma (one due to uveitis and one following corneal graft for acanthamoeba keratitis) and one case of combined extracapsular cataract extraction with trabeculectomy during which there was vitreous loss. In these cases a limbal based conjunctival flap was utilised and 5-FU soaked in a sponge was applied to the scleral bed, covered with the conjunctival flap, and left in place for 5 minutes, during which time the sponge was replenished with 5-FU at 1 minute intervals. 


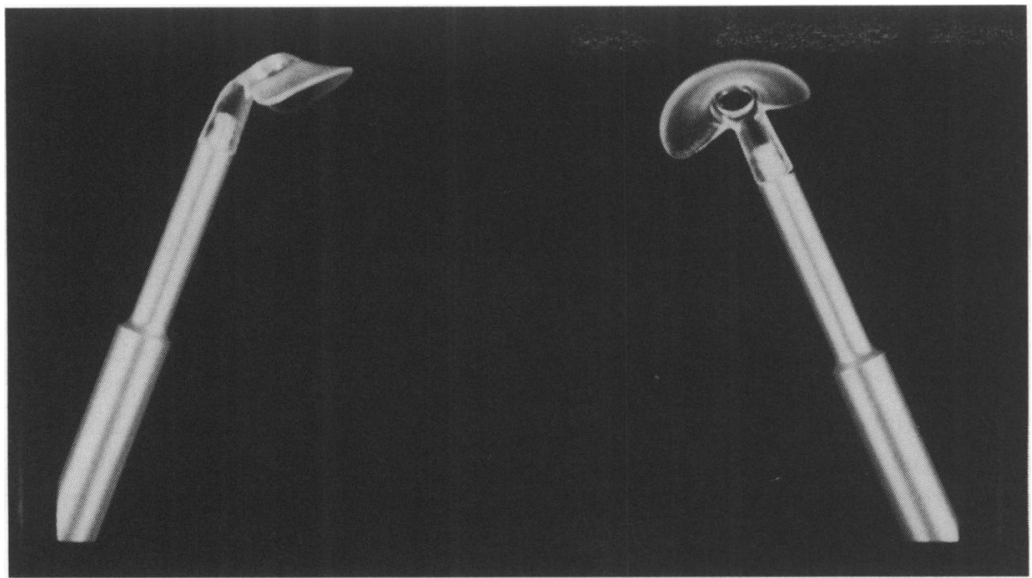

Figure 1 A Hoskins lens used for laser suture lysis.

Patients were only entered into the study if the following criteria were fulfilled: the IOP had to be $>21 \mathrm{~mm} \mathrm{Hg}$ in the presence of a deep quiet anterior chamber with no bleb formation following surgery. Gonioscopy was performed in all cases to exclude any blockage to the internal fistula. If these criteria were met it was assumed that aqueous outflow was obstructed as a result of an overtight scleral flap and suture lysis was attempted. All cases had lysis delayed for at least 48 hours to allow the conjunctival wound edges to heal and to allow for a trial period of ocular massage which was performed digitally four times a day through the upper lid on each side of the operation site.

Suture lysis was performed with the patient seated at a laser slit-lamp. The laser used was a crystal focus frequency doubled neodymium YAG (Nd:YAG) (Biovision, CA, USA) emitting monochromatic green light of wavelength $532 \mathrm{~nm}$. Benoxinate drops were instilled into the conjunctival sac and a Hoskins lens (Ocular Instruments, USA, Fig 1) gently applied to the superior part of the globe with the patient looking downwards. The upper lid was retracted using either the flange of the Hoskins lens or, if this was not feasible, an assistant held the upper lid open. The conjunctiva was compressed with the central glass button of the lens to visualise a tight nylon scleral flap suture which was brought into sharp focus and one to two laser shots applied to lyse the monofilament nylon. The spot size used was $100 \mu \mathrm{m}$, with a duration of 0.1 seconds, the only variable being the power used which was, on average, $0.6 \mathrm{~W}$ (range $0.35-1.00 \mathrm{~W}$ ). If no bleb appeared spontaneously, the globe was gently massaged digitally or with a glass rod on each side of the scleral flap to promote aqueous drainage. The IOP was measured within 5 minutes of lysis. If at this stage there was still no bleb and a high IOP persisted then another nylon suture was cut with the laser. Patients were commenced on 2 hourly betamethasone/neomycin drops and were followed up every few days for the first 10 days and at 2-3 weekly intervals thereafter.

The number of sutures placed in the scleral flap was examined to determine if laser suture lysis was more commonly required among those patients who had three sutures placed

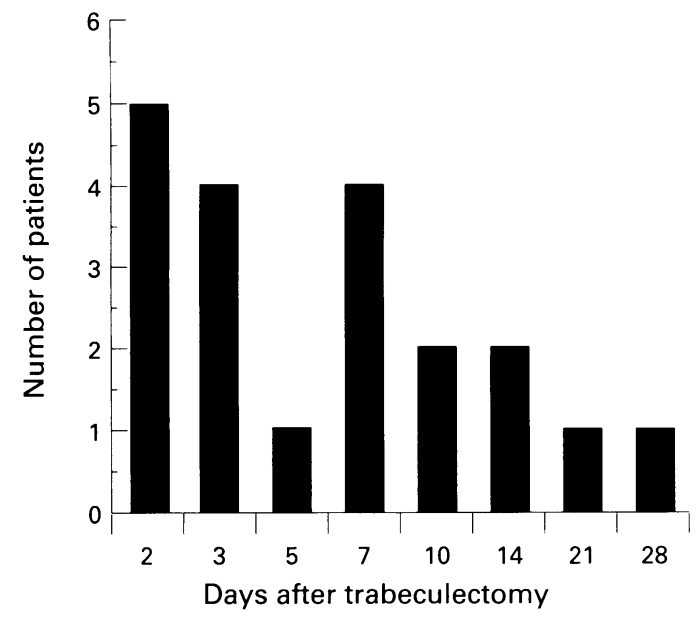

Figure 2 The timing of laser suture lysis in postoperative days.

compared with those with two sutures. A paired Student's $t$ test was used for the statistical analysis.

\section{Results}

Suture lysis was successful in all the cases (at least in the short term-that is, within 1 month). The median timing of suture lysis was 6 days post trabeculectomy surgery (range 2-28 days); however, most cases underwent lysis 2-3 days after drainage surgery (Fig 2). There were two exceptions; one patient underwent laser cutting 21 days and another 28 days after trabeculectomy. Thirteen out of the 20 cases $(65 \%)$ had three sutures placed in the rectangular scleral flap, the remaining seven having two. Five patients required lysis of two sutures before a bleb was formed with a lowered IOP, three of which had three sutures placed in the scleral flap. None of those patients with three sutures required lysis of all three sutures.

The mean pretrabeculectomy IOP was 31.0 (SD 6) $\mathrm{mm} \mathrm{Hg}$ and the mean post trabeculectomy IOP at the 6 month follow up was 15.8 (6.9) $\mathrm{mm} \mathrm{Hg}(p<0.0001)$. Mean IOP before suture lysis was 33.8 (8.9) $\mathrm{mm} \mathrm{Hg}$ and the immediate IOP 5 minutes after lysis was 13.2 $(7.9 \mathrm{~mm} \mathrm{Hg}(p<0.0001)$. Eighteen patients $(90 \%)$ had draining blebs and controlled IOP at the latest follow up (mean 14.1 (3.6) $\mathrm{mm}$ $\mathrm{Hg}$, in all cases IOP was less than $21 \mathrm{~mm} \mathrm{Hg}$ without medication). Two cases failed (IOP $>21 \mathrm{~mm} \mathrm{Hg}$ ) and required further medical or surgical treatment.

Complications after suture lysis are indicated in Table 1 . There were no cases of flat anterior chambers or ocular hypotony.

\section{Discussion}

The presence of a non-filtering bleb immediately after trabeculectomy surgery can pose

Table 1 Complications following laser suture lysis

\begin{tabular}{lll}
\hline Complication & Number & Outcome \\
\hline Conjunctival burn & 3 & No leak, settled spontaneously \\
Shallowing of AC & 3 & Settled spontaneously \\
Conjunctival flap & 1 & Settled with bandage contact \\
$\quad$ leak & 1 & lens \\
Cataract & 1 & Required phacoemulsification \\
Ocular hypotony & 0 & - \\
\hline
\end{tabular}


management difficulties for the ophthalmologist. Lack of aqueous outflow may be dealt with by surgical intervention and refashioning of a tight scleral flap. This is undesirable, however, in that it requires another formal surgical procedure for the patient which can lead to further inflammation, causing subconjunctival fibrosis and trabeculectomy failure. A less invasive method of enhancing drainage is to use preplaced releasable sutures and loosen these as the clinical situation demands. ${ }^{5}$ The latter technique depends upon adequate tying of releasable sutures in the first instance, and if these are too slack there is a greater chance of resulting ocular hypotony, shallow or flat anterior chambers which may again require further surgical intervention.

An alternative minimally invasive treatment strategy for an overtight scleral trapdoor is to cut or lyse taut sutures with a laser beam. This technique has been described with an argon laser as a means of safely controlling the early postoperative IOP. ${ }^{6-12}$ In the majority of cases the most substantial reduction in IOP occurs if laser suture lysis is performed within 2 weeks of surgery; however, it has been reported successfully when used up to 21 weeks postoperatively in cases of trabeculectomy with adjunctive therapy such as mitomycin $\mathrm{C}$ where wound healing is delayed. ${ }^{13} \mathrm{~A}$ recent retrospective study ${ }^{14}$ has failed to demonstrate a significant effect of laser suture lysis on the long term success rate of trabeculectomy although it is conceded that a randomised prospective trial is required to further evaluate its use. In addition, there are two reasons why this does not undermine the use of this procedure in the early postoperative period. Firstly, it allows the acute lowering of the IOP in situations when it is felt to be high enough to threaten the integrity of the optic nerve head and, secondly, it encourages the placement of a greater number and also tighter scleral flap sutures. This avoids the complications of excessive aqueous drainage in the early postoperative period and also affords the surgeon greater control of postoperative filtration by the selective and sequential release of sutures.

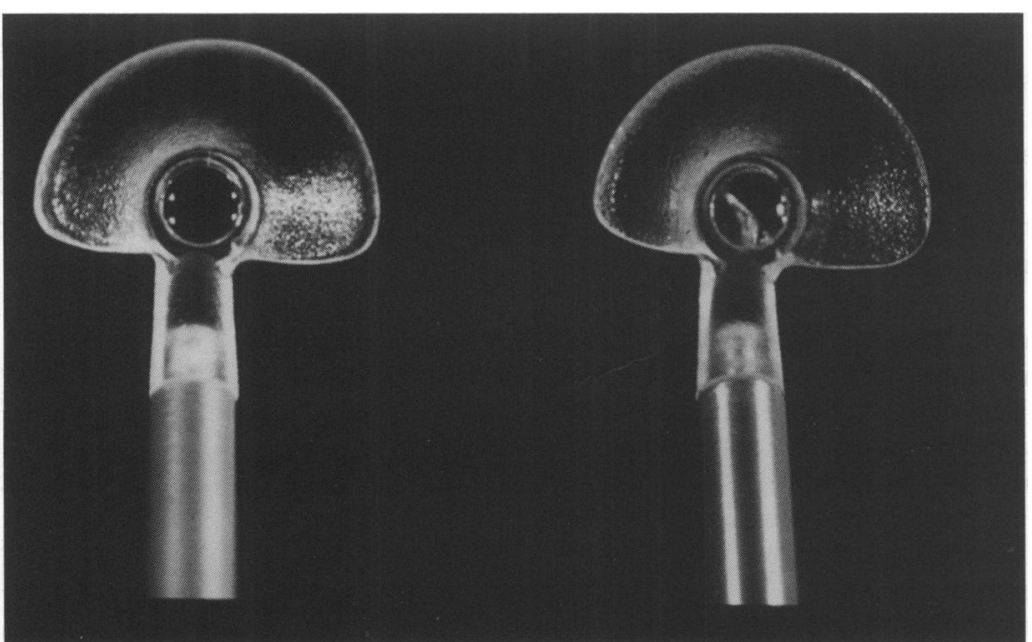

Figure 3 High power view of the head of a Hoskins lens to illustrate peripheral flange and a central focusing bulb. The lens on the right has been cracked after use with a neodymium YAG (1064 $\mathrm{nm})$ laser.
Melamed et $a l^{8}$ examined the use of laser suture lysis in combination with a tight, three suture scleral flap trabeculectomy. Not surprisingly non-drainage occurred in $73 \%$ of cases; however, after laser suture lysis of between one and three sutures, a success rate (IOP $<18 \mathrm{~mm}$ $\mathrm{Hg}$ ) of $91 \%$, in keeping with the findings of this study, was achieved. It has also been our experience that those patients with three scleral flap sutures have an increased risk of non-drainage, with $65 \%$ of the cases requiring laser lysis having three sutures as opposed to two. This is all the more significant when taking into consideration that the three suture technique is much less commonly performed by the surgeons in this unit. Despite this, all 13 of the three suture cases had a successful outcome (IOP $<21 \mathrm{~mm}$ $\mathrm{Hg}$, with no topical medication at latest follow up).

A Hoskins lens was used for focusing the laser onto black nylon sutures and the manufacturers of the lens also claim that a diode laser is a safe alternative source. A YAG laser (1064 $\mathrm{nm}$ ) can also be used; however, unless the focusing is accurate there is a risk of cracking the lens (Fig 3). We have utilised green light at a wavelength of $532 \mathrm{~nm}$ and found this to be extremely effective in lysing nylon sutures with no adverse effect on the glass button of the focusing device. Several different types of lens can be used for this procedure including the bulbous tip of a glass rod. ${ }^{6}{ }^{12}$ The main advantages of a Hoskins lens are that it has a flange which can be used to elevate the upper lid, and the centre glass button has a flat contour unlike a glass rod and therefore does not cause any optical distortion of the aiming beam (Figs 1 and 3). Pressure on the conjunctiva by the overlying lens causes thinning and blanching of the conjunctival vessels allowing the suture to be easily brought into focus even in the presence of subconjunctival haemorrhage and conjunctival oedema.

In this series the incidence of complications was found to be very low and those that occurred were relatively minor and self limiting. The three cases of conjunctival burns were superficial with no associated leak, and one case of aqueous leak from underneath the conjunctival flap settled with the application of a bandage contact lens. Three cases developed shallowing of the anterior chamber which settled spontaneously and there were no cases of ocular hypotony. The incidence of these complications can be minimised if the laser lysis is delayed for at least 48 hours, at which time there is already sufficient healing of the conjunctival flap to provide the necessary resistance to aqueous flow. ${ }^{8}$ One patient developed postoperative lens opacities requiring phacoemulsification with lens implant, although this was more likely to be associated with the trabeculectomy surgery than the laser suture lysis.

The two patients who had an unsuccessful surgical outcome were both high risk cases. The first case was a 37-year-old woman who developed secondary glaucoma following a penetrating keratoplasty for acanthamoeba keratitis. She had previously undergone a trab- 


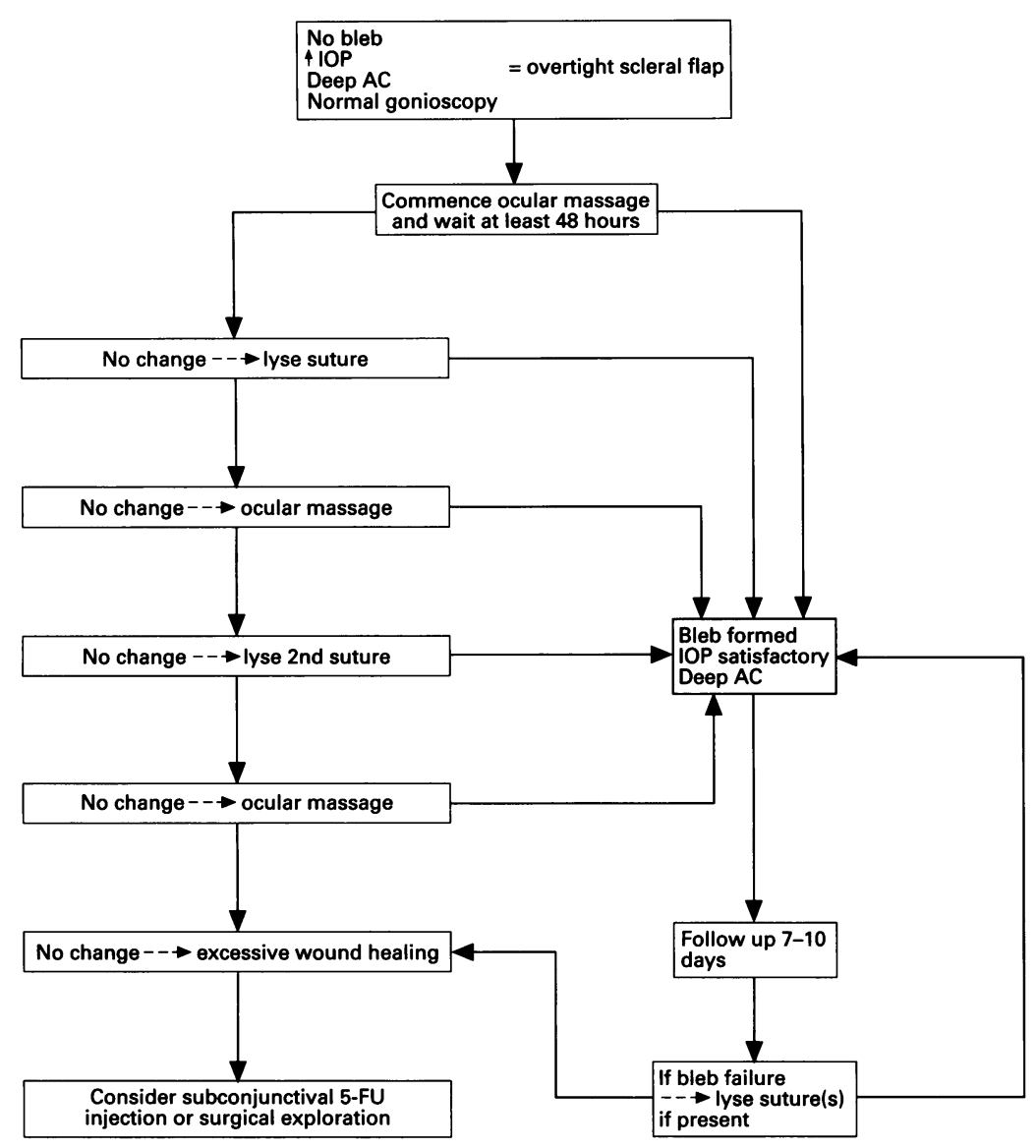

Figure 4 Algorithm for the early postoperative management of trabeculectomy bleb failure using laser suture lysis.
He subsequently underwent left YAG laser cyclocoagulation and the IOP remains controlled on topical $\beta$ blockers and twice daily oral acetazolamide.

In summary, the results of this study have shown that suture lysis using a frequency doubled crystal focus Nd:YAG laser is a safe and effective method of enhancing filtration after trabeculectomy. Figure 4 illustrates our algorithm for the early postoperative management of post trabeculectomy bleb failure using this technique. The critical importance of good drainage immediately after trabeculectomy has been shown recently. ${ }^{15}$ This study found that patients at risk of failure can be identified on the basis of IOP in the early postoperative period. In that report it was found that a successful outcome following trabeculectomy in terms of normalisation of IOP could be predicted with an accuracy of $92.6 \%$ if the IOP was $<17 \mathrm{~mm} \mathrm{Hg}$ on the first postoperative day. Likewise, a prediction of failure increased in accuracy with time, reaching $76 \%$ at 1 month. A high initial IOP $(>17 \mathrm{~mm} \mathrm{Hg})$ was an indicator that the procedure was more likely to fail and therefore such patients could be targeted for closer follow up. This emphasises the important role which laser suture lysis can contribute to surgical success. It has the advantage of being a simple technique which can be performed as an outpatient procedure, it does not require the acquisition of new surgical skills unlike the placement of releasable sutures, and avoids the potential anxiety to the patient and risks of bleb failure associated with surgical manipulation of scleral flap sutures.

further extraction. On this occasion a left trabeculectomy augmented with peroperative 5-FU was performed in the inferotemporal quadrant. The IOP remained controlled for the first week until the seventh postoperative day when it had risen to $30 \mathrm{~mm} \mathrm{Hg}$. Laser suture lysis was performed on both sutures with no apparent bleb formation and no reduction in the IOP. She was treated with hourly dexamethasone drops and on review 3 weeks later was found to have a pressure of $20 \mathrm{~mm} \mathrm{Hg}$ with a good bleb. Unfortunately, a further 6 weeks later the IOP had risen to $28 \mathrm{~mm} \mathrm{Hg}$ and she was started on betaxolol drops $0.5 \%$ twice daily. Eight months later the eye had become phthisical with an opaque vascularised graft.

The second patient was a 72 -year-old man with chronic angle closure glaucoma who developed malignant glaucoma after a left trabeculectomy. Five days postoperatively he underwent extracapsular cataract extraction with implant, refashioning of trabeculectomy, and reformation of anterior chamber. The IOP remained elevated despite full medical treatment (including acetazolamide) and a YAG laser capsulotomy was performed. Two weeks later, with the IOP measuring $36 \mathrm{~mm} \mathrm{Hg}$, a left laser suture lysis was performed on both scleral flap sutures resulting in formation of a bleb and a reduction of the IOP to $10 \mathrm{~mm} \mathrm{Hg}$. The following day the IOP had risen again to 35 $\mathrm{mm} \mathrm{Hg}$ and despite repeated injections of subconjunctival 5-FU the trabeculectomy failed.
The authors have no commercial interest in the equipment described herein.

1 Cairns JE. Trabeculectomy. Preliminary report of a new method. Am F Ophthalmol 1968;66:673-9.

2 Drance SM, Vargas E. Trabeculectomy and thermosclerectomy. A comparison of two procedures. Can 7 Ophthalmol 1973;8:413-5.

3 Blondeau P, Phelps CD. Trabeculectomy vs thermosclerostomy. A randomised prospective clinical trial. Arch stomy. A randomised prosp
Ophthalmol 1981;99:810-6.

4 Watkins FH, Brubaker RF. Comparison of partial thickness and full thickness filtration procedures in open angle glauand full thickness filtration procedures in

5 Kolker ME, Kass MA, Rait JL. Trabeculectomy with releasable sutures. Arch Ophthalmol 1994;112:62-6.

6 Lieberman MF. Suture lysis by laser and gonio lens. $A m \mathfrak{J}$ Ophthalmol 1983;95:257-8.

7 Hoskins HD, Migliazzo C. Management of failing filtering blebs with the argon laser. Ophthalmic Surg 1984;15:731-3.

8 Melamed S, Ashkenazi I, Glovinski J, Blumenthal M. Tight scleral flap trabeculectomy with post operative laser surure lysis. Am f Ophthalmol 1990;109:303-9.

9 Savage JA, Condon GP, Lytle RA, Simmons RJ. Laser suture lysis after trabeculectomy. Ophthalmology 1988;95: 1631-7.

10 Osher RH, Cohen JS. Management of acute post operative intraocular pressure elevation by laser induced filtration. $\mathcal{f}$ Cataract Refract Surg 1986;12:77-8. argon laser suture lysis after trabeculectomy. Am $\mathcal{F} O$ phthal

12 Menage MJ. Use of a glass rod in argon laser suture cutting after trabeculectomy. Eye 1993;7:599.

13 Pappa KS, Derick RJ, Weber PA, Kepetansky FM, Baker ND, Lehmann DM. Late argon laser suture lysis after mitomycin C trabeculectomy. Ophthalmology 1993;100: 1268-71.

14 Asamoto A, Yablonski ME, Matsushita M. A retrospective study of the effects of laser suture lysis on the long-term results of trabeculectomy. Ophthalmic Surg 1995;26:223-7.

15 Downes SM, Misson GP, Jones HS, O'Neill EC. The predictive value of postoperative intraocular pressures following trabeculectomy. Eye 1994;8:394-7.
11 Haynes WL, Alward WLM, McKinney JK. Low energy mol 1994;117:800-1. 